\title{
Lung Tissue Alterations were Size-dependent with Smaller Ones Induced More Effects and Related with Time Exposure of Gold Nanoparticles
}

\section{Mohamed Anwar K Abdelhalim*}

Department of Physics and Astronomy, College of Science, King Saud University, Saudi Arabia

\section{Abstract}

Background: The aim of the present study was to investigate the effects of intraperitoneal administration of GNPs on the histological alterations of the lung tissue of rats in an attempt to cover and understand the toxicity and the potential role of GNPs as a therapeutic and diagnostic tool.

Methods: A total of 40 healthy male Wistar-Kyoto rats were exposed to GNPs received $100 \mu$ l of GNPs infusion of 10,20 and $50 \mathrm{~nm}$ GNPs for 3 or 7 days. Animals were randomly divided into groups, 6 GNPs-treated rat groups and one control group (CG). Groups 1, 23 received infusion of $100 \mu \mathrm{l} \mathrm{GNPs}$ of size $10 \mathrm{~nm}$ (3 or 7 days), size 20 $\mathrm{nm}$ ( 3 or 7 days) and $50 \mathrm{~nm}$ ( 3 or 7 days), respectively.

Results: GNPs-treated rats receiving $100 \mu$ of 10 and $20 \mathrm{~nm}$ particles for 3 days demonstrated interstitial chronic inflammatory cell infiltration with extravasation of red blood cells and thickened alveolar wall and congested blood vessels while $50 \mathrm{~nm}$ GNPs-treated rat demonstrated interstitial inflammatory cells infiltrate with small lymphocytes, plasma cells and considerable number of eosinophils and surrounded by congested and dilated blood vessels. GNPs-treated rat which received $100 \mu \mathrm{l}$ of 10 and $20 \mathrm{~nm}$ particles for 7 days demonstrated diffuse interstitial lung pneumonia with small lymphocytes and plasma cells, considerable number of eosinophils, scattered extravasation of red blood cells surrounded by thickened, dilated and congested blood vessels while 50 nm GNPstreated rat demonstrated dense interstitial inflammatory cells infiltrate of chronic type.

Conclusions: The alterations induced by intraperitoneal administration of GNPs were size-dependent with smaller ones induced more affects and related with time exposure of GNPs. This study suggests that GNPs may interact with proteins and enzymes of the lung tissue interfering with the antioxidant defense mechanism and leading to reactive oxygen species (ROS) generation.

Keywords: Gold nanoparticles; Size; Lung tissue; Histology; Inflammatory; Nanotoxicity; Rats

\section{Introduction}

The NPs may differ in reactivity and solubility and may interact with all kinds of endogenous proteins, lipids, polysaccharides and cells. GNPs can easily enter cells and the demonstration that amine and thiol groups bind strongly to GNPs has enabled their surface modification with amino acids and proteins for biomedical applications $[1,2]$.

GNPs are thought also to be relatively non-cytotoxic [3] while the metallic nature of the metal derived NPs and the presence of transition metals encourages the production of reactive oxygen species (ROS) leading to oxidative stress [4,5]. There are differing reports of the extent of the toxic nature of these particles owing to the different modifications of the GNPs, surface functional attachments and shape and diameter size of the NPs $[6,7]$.

The particle size-dependent organ distribution of GNPs has been studied in vivo $[8,9]$. In vivo studies in rats exposed to aerosols of GNPs revealed that the NPs were rapidly taken into the system with the highest accumulation in the lungs, aorta, esophagus and olfactory bulb [10].

In order to understand and categorize the mechanisms for GNPs toxicity, information is needed on the response of living systems to the presence of GNPs of varying size, shape, surface, bulk chemical composition, and exposure duration. Very little information on these aspects is presently available and this implies that there is an urgent need for histological data for GNPs.

The histological and histochemical characterization in the lung tissues due to GNPs has not been documented and identified. In the present study, an attempt has been made to characterize the possible histological alterations in the lung tissues after intraperitoneal administration of GNPs and, if so, whether are related to the size of these GNPs and the time of exposure.

\section{Material and Methods}

A total of 40 healthy male Wistar-Kyoto rats were obtained from the Laboratory Animal Center (College of Pharmacy, King Saud University, Saudi Arabia). The rats nearly of the same age (12 weeks old) and weighing 220-240 g of King Saud University colony were used. Animals were randomly divided into groups, 3 GNPs-treated rats groups and one control group (CG). The 10, 20 and $50 \mathrm{~nm}$ GNPs were administered intraperitonealy at the rate of 3 or 7 days as follows: Group 1: received infusion of $100 \mu \mathrm{l}$ GNPs of size $10 \mathrm{~nm}$ for 3 or 7 days $(\mathrm{n}=10)$; Group 2: received infusion of $100 \mu \mathrm{l}$ GNPs of size $20 \mathrm{~nm}$ for 3

*Corresponding author: Dr. Mohamed Anwar K Abdelhalim, Department of Physics and Astronomy, College of Science, King Saud University, P.O. 2455, Riyadh 11451, Saudi Arabia, E-mail: abdelhalimmak@yahoo.com, mabdulhleem@ksu.edu.sa

Received May 06, 2012; Accepted June 20, 2012; Published June 22, 2012

Citation: Abdelhalim MAK (2012) Lung Tissue Alterations were Size-dependent with Smaller Ones Induced More Effects and Related with Time Exposure of Gold Nanoparticles. J Cancer Sci Ther 4: 170-173. doi:10.4172/1948-5956.1000135

Copyright: (c) 2012 Abdelhalim MAK. This is an open-access article distributed under the terms of the Creative Commons Attribution License, which permits unrestricted use, distribution, and reproduction in any medium, provided the original author and source are credited. 
or 7 days $(\mathrm{n}=10)$; Group 3: received infusion of $100 \mu \mathrm{l}$ GNPs of size 50 $\mathrm{nm}$ for 3 or 7 days $(\mathrm{n}=10)$. Control group: received no GNPs $(\mathrm{n}=10)$.

The rats were maintained on standard laboratory rodent diet pellets and housed in humidity and temperature-controlled ventilated cages on a $12 \mathrm{~h}$ day/night cycle. All experiments were conducted in accordance with the guidelines approved by King Saud University Local Animal Care and Use Committee.

Fresh portions of the lung from each rat were cut rapidly, fixed in neutral buffered formalin (10\%), then dehydrated, with grades of ethanol (70, 80, 90, 95 and 100\% ). Dehydration was then followed by clearing the samples in 2 changes of xylene. Samples were then impregnated with 2 changes of molten paraffin wax, then embedded and blocked out. Paraffin sections $(4-5 \mu \mathrm{m})$ were stained with hematoxylin and eosin (the conventional histological stain) according to Pearse [11]. The bright-field images were acquired using a Nikon Eclipse 800 microscope equipped with a Nikon DXM1200 color CCD camera (Nikon Instruments Inc., Melville, NY).

Stained sections of control and treated rats were examined for alterations in the lung tissues for the presence of lung pneumonia with small lymphocytes, plasma cells and eosinophils, extravasation of red blood cells, alveolar spaces, and congestion and dilation of blood vessels.

\section{Results and Discussion}

\section{Size and morphology of different gold nanoparticles (GNPs)}

The 10 and $20 \mathrm{~nm}$ GNPs show spherical shape while the $50 \mathrm{~nm}$ GNPs show hexagonal shape. The mean size for GNPs was calculated from the images taken by the transmission electron microscope (TEM): The $10 \mathrm{~nm}$ GNPs was of mean size $9.45 \pm 1.33 \mathrm{~nm}, 20 \mathrm{~nm}$ GNPs was of mean size $20.18 \pm 1.80$ and the $50 \mathrm{~nm}$ GNPs was of mean size 50.73 \pm 3.58 [12-15].

\section{Histological alterations}

No mortality occurred for the administration periods 3 and 7 days of GNPs in any of the experimental groups of the present investigation, and no alterations were observed in the appearance and behavior of GNPs treated rats in comparison with the control ones.

Control group (Figure 1): Microscopic pictures show GNPsnormal rat demonstrated well-formed and opened alveoli with its normal appearing septum, few scattered small lymphocytes and minimal eosinophils and with no other pathological changes.

In comparison with the control group, the following histological alterations were detected in the lung tissue of GNPs treated rats. These histological alterations were observed in Figures 2-7. The histological alterations can be summarized as follows:

1) GNPs-treated rat receiving $100 \mu \mathrm{l}$ of $10 \mathrm{~nm}$ particles for 3 days demonstrated interstitial chronic inflammatory cells infiltrate, considerable amount of scattered red blood cells with patent alveolar spaces, and thickened congested blood vessels as shown in Figure 2.

2) GNPs-treated rat receiving $100 \mu \mathrm{l}$ of $10 \mathrm{~nm}$ particles for 7 days demonstrated diffuse interstitial lung pneumonia with small lymphocytes and plasma cells, considerable number of eosinophils, scattered extravasation of red blood cells surrounded by thickened blood vessels, and patent alveolar spaces as shown in Figure 3.

3) GNPs-treated rat receiving $100 \mu \mathrm{l}$ of $20 \mathrm{~nm}$ particles for 3 days

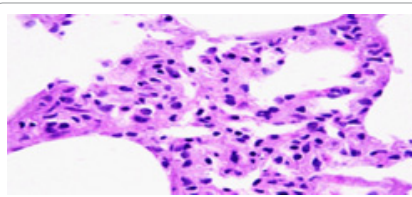

$(100 \times 2)$

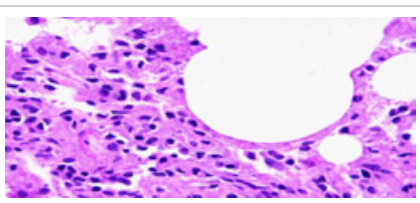

$(100 \times 7)$

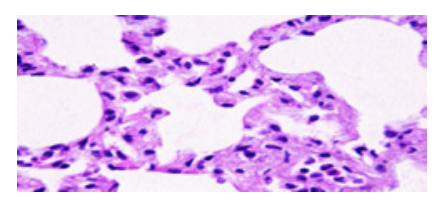

(100 x 5)

Figure 1: GNPs-normal rat demonstrated well-formed and opened alveoli with its normal appearing septum, few scattered small lymphocytes and minimal eosinophils, and with no other pathological changes.

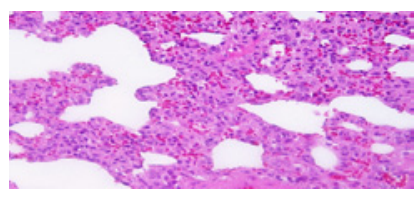

(40 $\mathbf{x 1 1 )}$

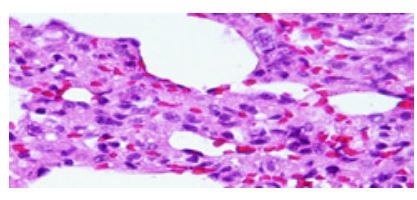

$(100 \times 5)$
Figure 2: GNPs-treated rat received $100 \mu$ of $10 \mathrm{~nm}$ particles for 3 days demonstrated inflammatory cells infiltrate with extravasation of red blood cells and with thickened alveolar wall.

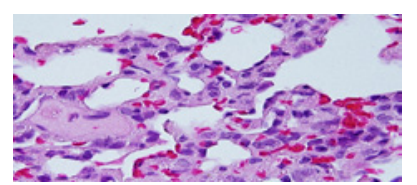

(100 $\times 5)$

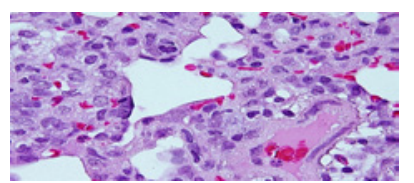

(100x4)
Figure 3: GNPs-treated rat received $100 \mu \mathrm{l}$ of $10 \mathrm{~nm}$ particles for 7 days demonstrated diffuse interstitial tissue infiltrated by chronic inflammatory cells, scattered red blood cells, patent alveolar spaces, and congested and dilated blood vessels.

demonstrated inflammatory cells infiltrate with extravasation of red blood cells and with thickened alveolar wall as shown in Figure 4.

4) GNPs-treated rat receiving $100 \mu \mathrm{l}$ of $20 \mathrm{~nm}$ particles for 7 days demonstrated diffuse interstitial tissue infiltrated by chronic inflammatory cells, scattered red blood cells, patent alveolar spaces, and congested and dilated blood vessels as shown in Figure 5.

5) GNPs-treated rat receiving $100 \mu \mathrm{l}$ of $50 \mathrm{~nm}$ particles for 3 days demonstrated interstitial inflammatory cells infiltrate with small lymphocytes, plasma cells and considerable number of eosinophils, and surrounded by congested and dilated blood vessels with patent clear alveolar spaces as shown in Figure 6.

6) GNPs-treated rat receiving $100 \mu \mathrm{l}$ of $50 \mathrm{~nm}$ particles for 7 days demonstrated dense interstitial inflammatory cells infiltrate of chronic type with patent clear alveolar space as shown in Figure 7.

The alterations induced by intraperitonealy administration of GNPs were size-dependent with smaller ones inducing more affects 
and related with time exposure of GNPs. None of the above alterations were observed in the lung tissue of any member of the control group.

This infiltration was more prominent after 7 days of administration and in rats receiving 10 and $20 \mathrm{~nm}$ GNPs than those receiving $50 \mathrm{~nm}$ GNPs. The appearance of interstitial lung pneumonia with small lymphocytes, plasma cells and eosinophils, extravasation of red blood cells, and congested, thickened and dilated blood vessels may suggest that GNPs could interfere with the antioxidant defense mechanism, leading to reactive oxygen species (ROS) generation which in turn may imitate an inflammatory response. Inflammatory cells infiltration was seen in the portal triads and the perioral zones of GNPs treated rats. The infiltrate cells were mainly lymphocytes and plasma cells [12-15].

It has been reported that $5 \mathrm{~nm}$ GNPs caused significantly greater oxidative stress and cytotoxicity effects than larger ones [16,17]. The $5 \mathrm{~nm}$ GNPs have shown to catalyze nitric oxide (NO) production

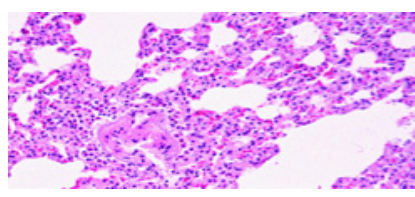

$(40 \times 1)$

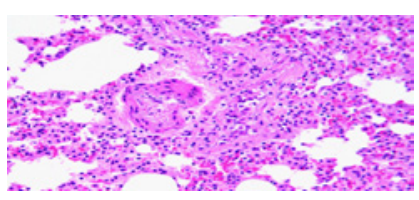

(40 $\times 3)$
Figure 4: (A-C) GNPs-treated rat received $100 \mu \mathrm{l}$ of $20 \mathrm{~nm}$ particles for 3 days demonstrated interstitial chronic inflammatory cells infiltrate, considerable amount of scattered red blood cells with patent alveolar spaces, and thickened congested blood vessels.

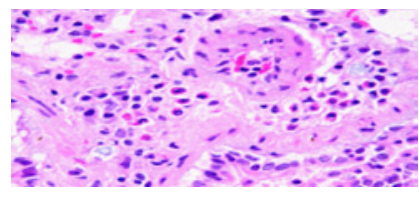

(100 $\times 8)$

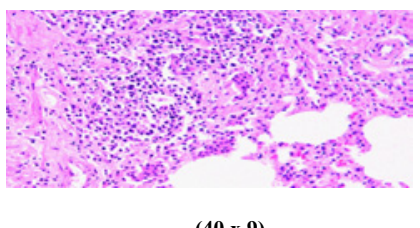

(40 x 9)
Figure 5: GNPs-treated rat received $100 \mu \mathrm{l}$ of $20 \mathrm{~nm}$ particles for 7 days demonstrated diffuse interstitial lung pneumonia with small lymphocytes and plasma cells, considerable number of eosinophils, scattered extravasation of red blood cells surrounded by thickened blood vessels, and patent alveolar spaces.

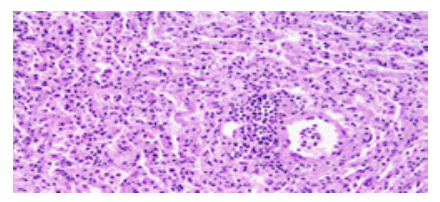

$(40 \times 2)$

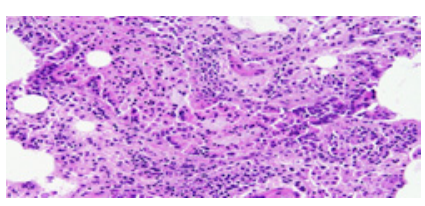

$(40 \times 6)$

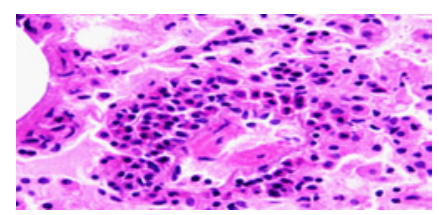

$(100 \times 7)$

Figure 6: GNPs-treated rat received $100 \mu \mathrm{l}$ of $50 \mathrm{~nm}$ particles for 3 days demonstrated interstitial inflammatory cells infiltrate with small lymphocytes, plasma cells and considerable number of eosinophils, and surrounded by congested and dilated blood vessels.

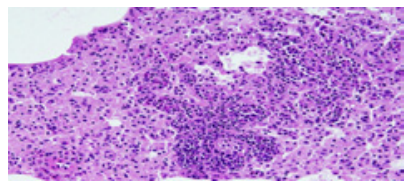

$(40 \times 5)$

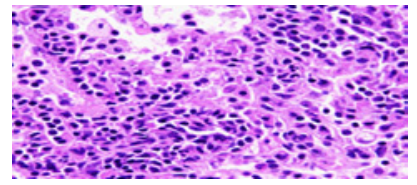

$(100 \times 1)$
Figure 7: GNPs-treated rat received $100 \mu \mathrm{l}$ of $50 \mathrm{~nm}$ particles for 7 days demonstrated dense interstitial inflammatory cells infiltrate of chronic type with patent clear alveolar space.

from endogenous S-nitroso adducts with thiol groups in blood serum. NO reacts rapidly with superoxide producing peroxynitrite $\left(\mathrm{ONOO}^{-}\right)$which can interact with lipids, DNA, and proteins via direct oxidative reactions or via indirect radical-mediated damage [17]. ROS production could result from the proportionately high surface area of GNPs used in this investigation [18].

NPs are nearly of same dimensions as some biological molecules such as proteins and nucleic acids. Many of these biomolecules consist of long macromolecular chains which are folded and shaped by cooperative and weak interaction between side groups. The GNPs may intrude into these complex folded structures.

GNPs activate the phagocytic activity of the sinusoidal cells by increasing the number of Kupffte cells to help in removing the accumulated GNPs where lysosomes are involved in the intracellular breakdown into small metabolic products. Kupffer cell hyperplasia is contributed to hepatic oxidative stress [19].

Fatty change was observed in some swelling hepatocytes of rats exposed to $100 \mu \mathrm{l}$ of $10 \mathrm{~nm}$ GNPs and to lesser extent in the ones exposed to larger particles. This hepatic liposis was more prominent in rats exposed to GNPs for 7 days than those receiving the treatment for 3 days [12-15]. Hepatocytes fatty change might be due to lipid peroxidation that leads to rough endoplasmic damage.

The rats receiving 10 and $20 \mathrm{~nm}$ GNPs showed lung pneumonia with small lymphocytes, plasma cells and eosinophils, extravasation of red blood cells, alveolar spaces, and congestion and dilation of blood vessels of red blood cells. Less disruption was observed in rats exposed to $50 \mathrm{~nm}$ GNPs while more damage was detected after 7 days than 3 days of GNPs exposure. This alteration might indicate lung tissue damage and congestion by GNPs exposure.

The interaction of NPs with living systems is also affected by the characteristic dimensions. As noted above, GNPs, of smaller size may reach inside biomolecules, a situation not possible for larger GNPs. It has been reported that inhaled NPs reach the blood and may reach other target sites such as the liver, lung or blood cells [20,21].

The reduction in particle size results in an enormous increase of surface to volume ratio, so relatively more molecules of the chemical are present on the surface, thus enhancing the intrinsic toxicity [21]. This may be one of the reasons that smaller GNPs are generally more toxic than larger particles of the same insoluble material when compared on a mass dose base [22].

For several different NPs, only Co induced toxicity in endothelial cells, which was accompanied by the production of the proinflammatory cytokine IL8 [23]. The $\mathrm{Cd}, \mathrm{Ni}$ and $\mathrm{Pb}$ concentrations significantly increased in blood and several tissues of rats after the intraperitoneal administration of 10, 20 and $50 \mathrm{~nm}$ GNPs compared 
Citation: Abdelhalim MAK (2012) Lung Tissue Alterations were Size-dependent with Smaller Ones Induced More Effects and Related with Time Exposure of Gold Nanoparticles. J Cancer Sci Ther 4: 170-173. doi:10.4172/1948-5956.1000135

with the control while different changes were observed with the Co concentrations [24].

The present study demonstrates that the inflammation produced in the lung tissue and other tissues/or organs [12-15] was more prominent with smaller GNPs inducing more affects and related with time exposure of GNPs.

In present study we have not measured GNPs concentration in urine and feces, but this point will be taken into our consideration in other new additional experiments.

This study suggests that additional experiments related to oxidative stress in cells and organs, plasma, tissues cytokine are now taken in consideration and performed to cover and understand the toxicity induced by administration of GNPs.

\section{Conclusions}

Histological alterations induced in the lung tissue exposure as shown from the results of the present work can be summarized as interstitial lung pneumonia with small lymphocytes, plasma cells and considerable number of eosinophils, extravasation of red blood cells, and congested, thickened and dilated blood vessels. One might conclude that these alterations are size-dependent with smaller ones inducing more damage in relation with the time of exposure of GNPs.

\section{Competing Interests}

Author declares that he has no competing interests.

\section{Author's contributions}

Abdelhalim MAK has analyzed data, interpreted and written the final draft of this manuscript. The animal model used in this study was obtained from the Laboratory Animal Center (College of Pharmacy, King Saud University, Saudi Arabia). Abdelhalim MAK has conceived the study and its design and obtained research grants for this study. The authors have read and approved the final manuscript.

\section{Acknowledgements}

The author is very grateful to the National Plan of Science and Technology (NPST). This research was financially supported by the National Science and Technology Innovation Plan (NSTIP), Research No. 08-ADV206-02 and Research No. 09-NAN670-02, College of Science, King Saud University, Saudi Arabia.

\section{References}

1. Connor EE, Mwamuka J, Gole A, Murphy CJ, Wyatt MD (2005) Gold nanoparticles are taken up by human cells but do not cause acute cytotoxicity. Small 1: 325-327.

2. Dani RK, Kang M, Kalita M, Smith PE, Bossmann SH, et al. (2008) MspA Porin-Gold Nanoparticle Assemblies: Enhanced Binding through a Controlled Cysteine Mutation. Nano Lett 8: 1229-1236.

3. MacNee W, Donaldson K (2003) Mechanism of lung injury caused by PM10 and ultrafine particles with special reference to COPD. Eur Respir J Suppl 40: 47S-51S.

4. Jia HY, Liu Y, Zhang XJ, Han L, Du LB, et al. (2009) Potential oxidative stress of gold nanoparticles by induced-NO releasing in serum. J Am Chem Soc 131: 40-41.

5. Kim SY, Lee YM, Baik DJ, Kang JS (2003) Toxic characteristics of methoxy poly(ethylene glycol)/poly(epsilon-caprolactone) nanospheres; in vitro and in vivo studies in the normal mice. Biomaterials 24: 55-63.

6. Takahashi H, Niidome Y, Niidome T, Kaneko K, Kawasaki H, et al. (2006)
Modification of gold nanorods using phosphatidylcholine to reduce cytotoxicity Langmuir 22: 2-5.

7. Pan Y, Neuss S, Leifert A, Fischler M, Wen F, et al. (2007) Size-dependent cytotoxicity of gold nanoparticles. Small 3: 1941-1949.

8. Barathmanikanth S, Kalishwaralal K, Sriram M, Pandian SR, Youn HS, et al. (2010) Anti-oxidant effect of gold nanoparticles restrains hyperglycemic conditions in diabetic mice. J Nanobiotechnology 8: 16.

9. Schrand AM, Braydich-Stolle LK, Schlager JJ, Dai L, Hussain SM (2008) Can silver nanoparticles be useful as potential biological labels? Nanotechnology 19: 235104

10. Lanone S, Boczkowski J (2006) Biomedical applications and potential health risks of nanomaterials: molecular mechanisms. Curr Mol Med 6: 651-663.

11. Pearse AE (1985) Histochemistry: Theoritical and applied. Analytical technology. (4th edn), Vol. 2. Churchill-Livingstone, Edinburgh.

12. Abdelhalim MA, Jarrar BM (2011) Gold nanoparticles administration induced prominent inflammatory, central vein intima disruption, fatty change and Kupffer cells hyperplasia. Lipids Health Dis 10: 133.

13. Abdelhalim MA, Jarrar BM (2011) The appearance of renal cells cytoplasmic degeneration and nuclear destruction might be an indication of GNPs toxicity. Lipids Health Dis 10: 147

14. Abdelhalim MA, Jarrar BM (2011) Renal tissue alterations were size-dependent with smaller ones induced more effects and related with time exposure of gold nanoparticles. Lipids Health Dis 10: 163

15. Abdelhalim MA, Jarrar BM (2011) Gold nanoparticles induced cloudy swelling to hydropic degeneration, cytoplasmic hyaline vacuolation, polymorphism, binucleation, karyopyknosis, karyolysis, karyorrhexis and necrosis in the liver Lipids Health Dis 10: 166

16. Pan Y, Leifert A, Ruau D, Neuss S, Bornemann J, et al. (2009) Gold nanoparticles of diameter $1.4 \mathrm{~nm}$ trigger necrosis by oxidative stress and mitochondrial damage. Small 5: 2067-2076.

17. Lasagna-Reeves C, Gonzalez-Romero D, Barria MA, Olmedo I, Clos A, et al. (2010) Bioaccumulation and toxicity of gold nanoparticles after repeated administration in mice. Biochem Biophys Res Commun 393: 649-655.

18. Nel A, Xia T, Mädler L, Li N (2006) Toxic potential of materials at the nanolevel. Science 311: 622-627.

19. Neyrinck A (2004) Modulation of Kupffer cell activity: physio-pathological consequences on hepatic metabolism. Bull Mem Acad R Med Belg 159: 358366 .

20. Del Monte U (2005) Swelling of hepatocytes injured by oxidative stress suggests pathological changes related to macromolecular crowding. Med Hypotheses 64: 818-825

21. Oberdörster G, Sharp Z, Atudorei V, Elder A, Gelein R, et al. (2002) Extrapulmonary translocation of ultrafine carbon particles following whole-body inhalation exposure of rats. J Toxicol Environ Health A 65: 1531-1543.

22. Kreyling WG, Semmler M, Erbe F, Mayer P, Takenaka S, et al. (2002) Translocation of ultrafine insoluble iridium particles from lung epithelium to extrapulmonary organs is size dependent but very low. J Toxicol Environ Health A 65: 1513-1530.

23. Oberdörster G, Finkelstein JN, Johnston C, Gelein R, Cox C, et al. (2000) Acute pulmonary effects of ultrafine particles in rats and mice. Res Rep Health Eff Inst (96): 5-74; disc 75-86.

24. Abdelhalim MK, Al-Ayed MS, Moussa SA (2011) Elucidation the response of heavy elements levels to intraperitoneal administration of different gold nanoparticles into rats for period of 3 days in vivo. African Journal of Microbiology Research 5: 3267-3272. 